\title{
Field Comparison between Blood Immunochromatographic Card Test and Microscopy in Diagnosis of Bancroftian Filariasis in Northern Taraba State, Nigeria
}

\author{
Elkanah $O S^{1}$, Elkanah $D S^{1}$, Wahedi $J^{2}{ }^{*}$, Kela $S L^{3}$, Samaila $A B^{1}$ and Madara $A A^{4}$ \\ ${ }^{1}$ Department of Biological Sciences, Faculty of Natural Sciences, Taraba State University, Nigeria \\ ${ }^{2}$ Department of Zoology, Faculty of Science, Adamawa State University, Nigeria \\ ${ }^{3}$ Applied Parasitology Unit, Department of Biological Science, ATBU Bauchi, Nigeria \\ ${ }^{4}$ Department of Biological Sciences, Faculty of Sciences, University of Abuja, Nigeria
}

*Corresponding author: Wahedi JA, Department of Zoology, Faculty of Science, Adamawa State University, Mubi, P.M.B 25, Adamawa State, Nigeria

\begin{abstract}
Diagnosis of filariasis is important for Programme Managers and Physicians. This study was undertaken to determine the prevalence of lymphatic filariasis in eleven communities of Northern Taraba State. The study employed standard parasitological techniques for diagnosis, and the use of Immunochromatographic Card Test (ICT) to detect Circulating Filarial Antigen (CFA). Chi Square was used to compare and test for differences in infections among communities. 294 persons were examined, and $31.29 \%$ were confirmed positive for Wuchereria bancrofti. The performance of ICT card compared with Thick Blood Film microscopy was commendable. Thick Blood Film/microscopy identified $26.19 \%$ infection of the population, while the ICT card detected $31.29 \%$ CFA from the same population. The prevalence of CFA differ significantly $(p<0.05)$, and no significant difference between antigenaemia in males and females. However, chi-square test revealed a significant difference in the prevalence of antigenaemia among the different age groups. Antigenaemia was detected in 92 persons, and 34 (36.96\%) persons are both antigenaemia positive and microfilaria positive, while 58 persons $(63.04 \%)$ with antigenaemia but without microfilaraemia. Of the 202 negative subjects $43(21.29 \%)$ had microfilaria in their blood samples, while 159 (78.71\%) were negative for microfilaraemia. The ability of the ICT to accurately identify all those with the disease was $44.16 \%$, while the ability of the ICT to sort out those without disease was $73.27 \%$. The Positive Predictive Value was $36.96 \%$, while the Negative predictive value was $78.71 \%$ burden of LF. In conclusion, since the ICT had identified $31.29 \%$ people with LF as against $26.19 \%$ by Microscopy, it was suggested that it could be employed in mass survey for Lymphatic filariasis to institute intervention activities in the area.
\end{abstract}

\section{Keywords}

Bancroftian filariasis, ICT, Thick blood film, Sensitivity, Specificity

\section{Introduction}

More than 100 million individuals worldwide are estimated to suffer from lymphatic filariasis (LF) caused by infection with the mosquito-borne filarial nematode, Wuchereria bancrofti $[1,2]$. Available literatures on the status of the disease in Nigeria show that the disease is prevalent and widespread in the six geo-political zones of the country $[3,4]$. Nigeria is the third most endemic country in the world for LF after India and Indonesia $[5,6]$. WHO [7] identified LF as the second leading cause of permanent and long-term disability and a major contributor to poverty in the world. In Benue State, Nigeria, rapid epidemiological and social-cultural assessment lymphatic filariasis amongst the Igede ethnic group was conducted [8].

Diagnosis of filariasis is important for programme managers for situation analysis, for monitoring and evaluation of intervention measures and for physicians in case detection and treatment/management [9]. However, diagnosis of bancroftian filariasis requires night time parasitological examination because the microfilariae appear in peripheral blood highest in number at

Citation: Elkanah OS, Elkanah DS, Wahedi JA, Kela SL, Samaila AB, et al. (2020) Field Comparison between Blood Immunochromatographic Card Test and Microscopy in Diagnosis of Bancroftian Filariasis in Northern Taraba State, Nigeria. Int J Trop Dis 3:042. doi.org/10.23937/2643-461X/1710042 Accepted: December 22, 2020; Published: December 24, 2020

Copyright: (c) 2020 Elkanah OS, et al. This is an open-access article distributed under the terms of the Creative Commons Attribution License, which permits unrestricted use, distribution, and reproduction in any medium, provided the original author and source are credited. 
night and few or none during day time [10]. During the day, the microfilariae are in the lungs; at least two circadian rhythms are involved including, one in the microfilaria and the other, a property of the physiologic rhythm of the host [11]. Usually, thick blood samples are prepared from the night blood samples collected, allowed to dry overnight or air dried could be stained Geimsa or haematoxylin. The stains allow morphological identification of filarial species. The diagnosis of this nocturnal periodic parasite is faced with several constraints including the belief that only witches take blood at night and the sacrosanct regard about it is a major setback in the diagnosis of bancroftian filariasis in Nigeria. In addition, women in Northern Nigeria are not permitted to leave their household at night, thus putting constraints on accurate diagnosis and reporting. These constraints have led to increasing quest for the most acceptable methods of diagnosis.

The use of ICT has been recommended as a rapid tool for defining the prevalence and distribution of filariasis as part of the global programme to eliminate lymphatic filariasis [12]. Galvaz Tan [13] stated that lymphatic filariasis mapping using ICT and community reporting of hydrocoel and elephantiasis increase access of the poor to diagnostic services and is a means of poverty alleviation. Gyapong, et al. [14] in a multi-country study (Benin, Burkina Faso, Ghana and Togo) employed the rapid geographical assessment of filariasis in Africa (RAGFIL) using the ICT tests. Therefore, as now the reliability of the use of immunochromatographic card test to identify infected persons with bancroftian filariasis in Savannah ecological zones need to be understood.

It was in the light of this that this study was designed to determine the performance of immunochromatographic card test status of the disease in Northern Taraba State with the view of enriching the epidemiological baseline data in Nigeria.

\section{Materials and Methods}

\section{Description of the study area}

The study was performed in Taraba State. It lies approximately between Latitude $6025^{\prime}$ and $9030^{\prime} \mathrm{N}$ and Longitude $9030^{\prime}$ and $11045^{\prime} \mathrm{E}$. It is bordered on the West by Nassarawa and Plateau States, South East by Bauchi and Gombe States, North East by Adamawa State and South west by Benue State. Taraba State is bordered to the East by the Federal Republic of Cameroon which is an international boundary. The State has a land area of about $60,291 \mathrm{sq} . \mathrm{km}$, with a total population of 2,300,736 persons based on the 2006 National Census Figures. About $43,453 \mathrm{~km}^{2}$ of Taraba State is plain land, while $12,929 \mathrm{~km}^{2}$ is highland. The remaining 3,909 $\mathrm{km}^{2}$ is wetland. Majority of the people in the State are living in rural settlements consisting of hamlets and villages. Most of the inhabitants also live in rural agricultural areas with farming as the major occupation. Parts of the State have numerous streams traversing villages/ communities and draining into the major river Benue. Communities rely mainly on the streams and rivers for water supply. Domestic water is usually stored in and around homes in drums, clay pots and all sorts of metal and plastic containers which provide permanent breeding sites for mosquitoes and ecological associates [15].

\section{Ethical clearance and permission}

The study received ethical clearance certificate from an Institutional Health Research Committee and ethical approval of Taraba State Ministry of Health. Also, additional permission were sought and obtained from Local Government chairmen, Primary Health Care (PHC) department, Districts heads, Village heads, and key informants before the study commenced.

\section{Rapid assessment method}

On the scheduled day, informed oral consent of individuals who gathered at the agreed venue (village head compound, school or church premises) were sought and obtained after the explanation of the procedures and the benefits of the study before they were examined in secrecy for chronic clinical signs and symptoms of filariasis by the criteria of Nwoke, et al. [15] and Edungbola, et al. [16]. Clinical symptoms, lymphoedema of limbs, breast and scrotal elephantiasis were recorded in personal data form containing the patients' name. Female examinations were restricted to the legs, arms and breast because of cultural inhibitions in most communities.

\section{Parasitological examination}

Night blood samples of consenting individuals were obtained between $20.00 \mathrm{hrs}$ and $01.00 \mathrm{hrs}$. At each blood collection, the left thumb finger was cleaned with methylated spirit soaked in cotton wool. A sterile blood lancet was used to prick the finger and $60 \mu$ of blood collected on a slide was used to make a thick blood film which was air-dried and stained with $10 \%$ Giemsa solution for 10 minutes [17]. The slides were then examined under a light microscope at $\times 10, \times 40$ and $\times 100$ objective lenses. Sheathed microfilariae without caudal nuclei were classified as Wuchereria bancrofti [17].

\section{Method of ICT filariasis tests}

The collection of blood was carried out between 8:00 am and 6:00 pm in the day time at a designated venue agreed upon by members of the community. The finger prick method described by Cheesbrough [17] was employed. The patient's left index finger was cleaned with methylated spirit and then punctured using a sterile lancet. The pipette dropper enclosed in the ICT kit was used to suck $60 \mu \mathrm{l}$ of blood and applied into the sample well in the device (cassette) making sure that air bubbles were removed. Thereafter a drop of sample diluents contained in the kit was added immediately and 
Table 1: Distribution of $W$. bancrofti antigenaemia in 11 communities in the study area.

\begin{tabular}{|l|l|l|l|}
\hline Community & No. examined & No. Positive for ICT (\%) & Number positive for Microscopy (\%) \\
\hline Dankodi & 21 & $9(42.8)$ & $7(33.3)$ \\
\hline Garin Bako & 43 & $11(25.5)$ & $9(20.9)$ \\
\hline Janibanbum & 38 & $11(28.9)$ & $7(18.4)$ \\
\hline Jauro Ishaya & 28 & $10(35.7)$ & $8(28.5)$ \\
\hline Jauro Votto & 22 & $7(31.8)$ & $8(36.4)$ \\
\hline Lube & 37 & $10(27.6)$ & $8(21.6)$ \\
\hline Murbai & 25 & $9(36.0)$ & $7(28.0)$ \\
\hline Nwagbala & 21 & $10(47.6)$ & $8(38.1)$ \\
\hline Sembe & 11 & $2(18.2)$ & $2(18.2)$ \\
\hline Sobai & 20 & $4(20.0)$ & $3(15.0)$ \\
\hline Vango & 28 & $9(32.1)$ & $10(35.7)$ \\
\hline Total & 294 & $92(31.3)$ & $77(26.2)$ \\
\hline
\end{tabular}

Table 2: Comparison of performance of ICT as rapid screening test of LF and the true status of disease as determined by Thick Blood Film/Microscopy.

\begin{tabular}{|c|c|c|c|}
\hline Test Positive & True Positive $\left(\mathrm{T}_{\mathrm{p}}\right)$ & False Positive $\left(F_{p}\right)$ & PPV \\
\hline 77 & $34(36.96 \%)$ & $43(21.29 \%)$ & $\begin{array}{l}=\left(T_{P}\right) /\left(T_{P}\right)+\left(F_{P}\right) \\
=0.442\end{array}$ \\
\hline Test Negative & False Negative $\left(F_{p}\right)$ & True Negative $\left(T_{N}\right)$ & NPV \\
\hline \multirow[t]{2}{*}{217} & $58(63.04 \%)$ & $159(78.71 \%)$ & $\begin{array}{l}=\left(T_{N}\right) /\left(F_{N}\right)+\left(T_{N}\right) \\
=0.733\end{array}$ \\
\hline & $\begin{array}{l}\text { Sensitivity }=\left(T_{P}\right) /\left(T_{P}\right)+\left(F_{N}\right) \\
=0.369(44.16 \%)\end{array}$ & $\begin{array}{l}\text { Specificity }=\left(T_{N}\right) /\left(F_{P}\right)+\left(T_{N}\right) \\
=0.787(73.27 \%)\end{array}$ & \\
\hline
\end{tabular}

the specimen migrated by capillary action across the cassette. The test was read as positive if the test band/ line and ' $C$ ' band/line were seen in the viewing window. Any visible line on the test area indicated a positive test result even if it was lighter or darker than the ' $C$ ' line. The test was regarded as negative if only the ' $C$ ' line was seen. Positive results were apparent after 1-5 minutes. To ensure that no positive sample was deemed negative if it indicated negative after 10 minutes from the time the diluents was applied.

\section{Statistical analysis}

Data obtained was subjected to Chi Square analysis to compare and test for differences in infections among communities, using statistical package Epi- info software version 7.0. The diagnostic performance of immunochromatographic card test was assessed by calculating sensitivity, specificity, positive and negative predictive values using the formula of Chocrane [18]. The calculation was rechecked using Graph Prism 5.04 for confidence intervals as follows:

$$
\text { Sensitivity }=\frac{\left(T_{P}\right)}{\left(T_{P}\right)+\left(F_{N}\right)}
$$

Where: $\quad T_{\mathrm{P}}=$ True positive

$$
\mathrm{F}_{\mathrm{N}}=\text { False negative }
$$

$$
\text { Specificity }=\frac{\left(T_{N}\right)}{\left(F_{P}\right)+\left(T_{N}\right)}
$$

Where: $\quad T_{N}=$ True negative

$$
\mathrm{F}_{\mathrm{P}}=\text { False positive }
$$

Positive Predictive Value $(P P V)=\frac{\left(T_{P}\right)}{\left(T_{P}\right)+\left(F_{P}\right)}$

Negative Predictive Value $(N P V)=\frac{\left(T_{N}\right)}{\left(F_{N}\right)+\left(T_{N}\right)}$

\section{Results}

Of the 294 person examined $31.29 \%$ of the subjects were positive for $W$. bancrofti circulating filarial antigen (Table 1). The circulating filarial antigen prevalence among the communities differ significantly $(p<0.05)$ with no significant difference (in infection) between antigenaemia positive males and female $\left(\chi^{2}=0.491, \mathrm{df} 1, \mathrm{p}\right.$ $>0.05$ ) (Figure 1). However, Chi-square analysis showed a significant difference in the prevalence of antigenaemia among the different age group $\left(\chi^{2}=11.424, d f 5, p\right.$ $<0.05)$.

Table 2 compares the use of ICT test as rapid test to the true disease status as determine by microscopy and validation of ICT card as a screening test for detection of 


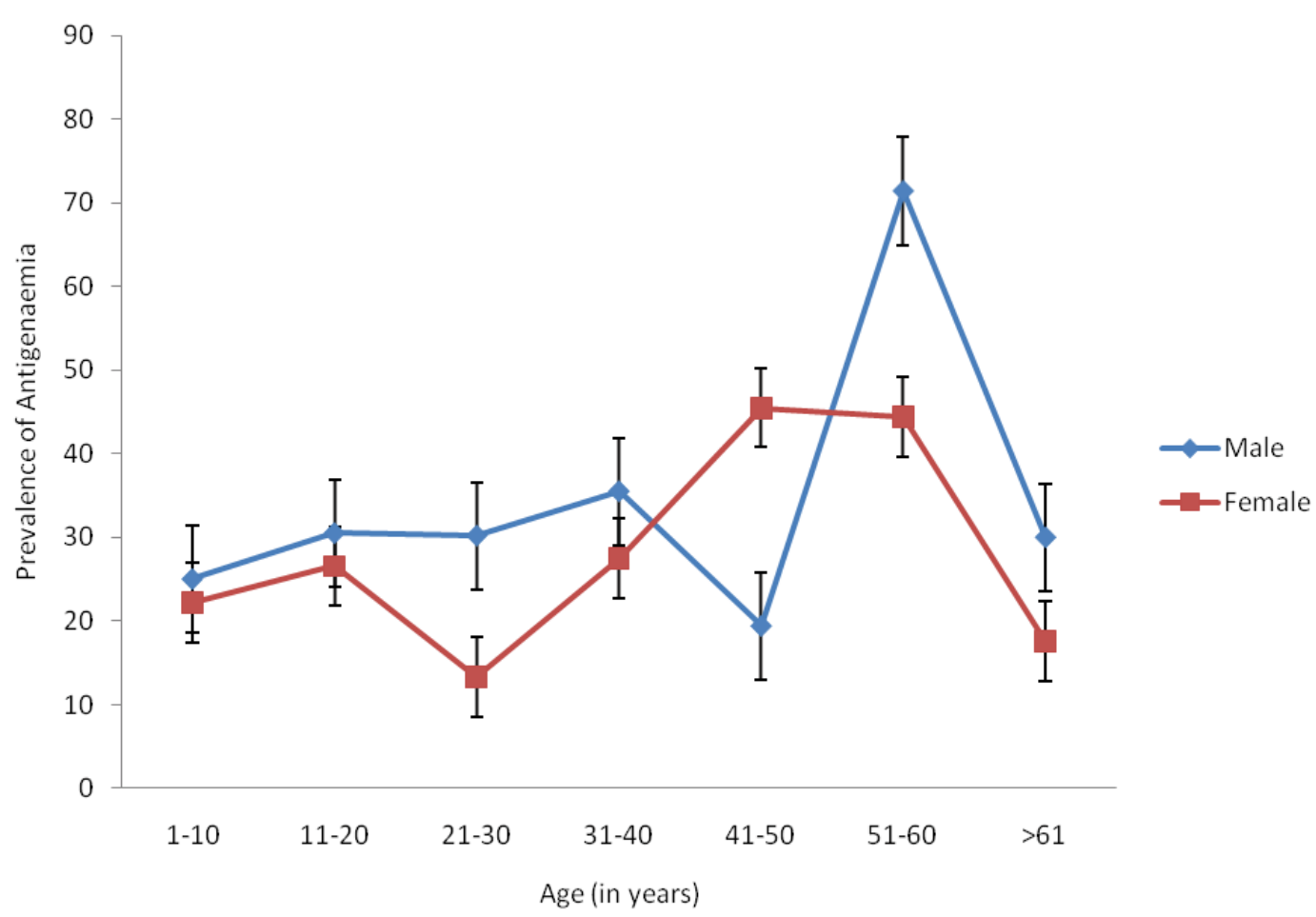

Figure 1: Prevalence of antigenaemia according to age and sex in the study area.

W. bancrofti in the study area. The result showed that 92 persons were detected with antigenaemia. Out of the number detected, 34 (36.96\%) persons were both antigenaemia positive and microfilaria positive (True positive) and 58 persons (63.04) with antigenaemia but without microfilaraemia (False Negative). The number detected negative for antigenaemia was 202, out of which 43 (21.29\%) had microfilaria in their blood samples (False positive) while 159 (78.71\%) of them are negative for microfilaraemia (True negative). The ability of the ICT to accurately identify all those with the disease (i.e. sensitivity) was $44.16 \%$ while the ability of the ICT to sort out all those without the disease (i.e. specificity) was $73.27 \%$. The Positive Predictive Value was $36.96 \%$ while the Negative Predictive Value was $78.71 \%$ burden of LF (Table 2).

\section{Discussion}

A sound knowledge of the distribution of any disease is a pre-requisite to planning effective control measures of that disease. This study provides reliable estimates of the prevalence and burden of lymphatic filariasis in parts of Muri Emirate council. The overall prevalence of filarial antigenaemia of $31.29 \%$ among individuals in the Northern Taraba communities even after at least one cycle of MDA in some of the communities has further confirmed that bancroftian filariasis is widely distributed in Taraba State. The high level of antigenaemmia observed here is comparable to that of Braga, et al. [19] in Brazil, and Iqbal and Sher [20] in Kuwait. However, the prevalence of CFA in the present study was much higher than that of $6.45 \%$ observed by Targema, et al. [21] in Benue State of Nigeria and $21.13 \%$ recorded in Afikpo North Ebonyi State Eastern Nigeria by Ngele and Adewale [22].
The high endemicity in these communities may be as a result of the availability of favourable mosquito breeding sites and deteriorating sanitary conditions [23]. In the current study, the prevalence of circulating filarial antigen was highest among subjects aged between 50-60 years. This is consistent with the observation of Ngele and Adewale [22] who recorded same among age group of 58-62. This however, contrasts the finding of Targema, et al. [21] who recorded higher antigenaemia among the $40-49$ years subjects. The ICT procedure was easy to use in the field and also very specific in detecting Circulating Filarial Antigen (CFA). It is indeed a great advancement in the diagnosis of bancroftian filariasis as compared to thick blood film preparation which is not convenient because of night blood collection [24,25].

The performance of immunochromatographic card has been evaluated in many temperate ecological zones $[19,26,27]$. However, in this study, $63.0 \%$ of person that were negative by the thick Blood film method seems to be positive in the ICT card test in the endemic areas. This is consistent with the findings of Braga, et al. [19] and Iqbal and Sher [20], who stated that the ICT card test, in addition to being able to detect microfilaraemia infection, probably identifies early stages of infections and single sex infections. Another possibility is that a low level of adult worm infection produces antigenaemia but no detectable microfilaraemia $[6,28]$. There is also the possibility since the evaluation of the test in the non-endemic area revealed a considerable number of examinations showing faint lines that following manufacturer's guide line were interpreted positive.

On the other hand, $21.2 \%$ individuals were positive by the thick blood smear appeared to be negative in the 
ICT card test. These findings raised concern about the specificity of the ICT whole blood card test or suggest a decrease sensitivity of the whole blood card test in cases with low microfilaraemia density. The low sensitivity of the ICT was reported previously from India in cases with low mf density [29]. Similarly, Koyandun, et al. [30] reported a significant decrease in Circulating Filarial Antigen (CFA) at 18 months after mass drug administration among the Myanmar migrants in Thailand. This might be the possible explanation to the above observations in the study area.

The performance of ICT card in comparison to Thick Blood Film microscopy in the present study is commendable. Thick Blood Film/microscopy identified $26.19 \%$ infection of the population, the ICT card detected CFA from $31.29 \%$ of the same population. This is consistent with the findings of Braga, et al. [19] who observed higher antigenaemia in comparison to microfilaraemia in Brazil. The $44.16 \%$ sensitivity and $73.27 \%$ of specificity recorded in this study is higher than the low sensitivity reported by Sunish, et al. [29] in India. However, the sensitivity observed in this study is lower than the $93.8 \%$ sensitivity recorded by lqbal and Sher [20] among migrant workers in Kuwait. Since the card requires minimal laboratory and technical support compared to microscopy and yield results in few minutes on the spot, it is thus more suitable for diagnosis of filarial infection in clinical laboratories and field survey.

The use of ICT card for mapping endemic communities has been recommended by Brooker and Utzinger [31] in order to target Mass Drug Administration. However, large scale field use of ICT card test in control programmes in endemic communities will require assessment of its cost and suitability. A cost analysis of the ICT card test and Thick Blood Smear Method were carried out in Sri Lanka [19]. The thick Blood Smear method was shown to have a lower price [ICT cost per unit US\$ 2.75 while TBS cost per unit - US\$ 0.30] [26]. However, certain features in the ICT card test proved to be extremely advantageous; high sensitivity, the ability to offer prompt diagnosis, no need for specialized technicians. These combined characteristics overcame the lowest price of thick blood smear method, making to be overall more cost effective option thereby justifying its use as a diagnostic tool in screening in endemic areas [19].

\section{Conclusion}

In conclusion, the use of ICT had identified $31.29 \%$ while Microscopy identified $26.19 \%$ of the same population with the disease. Thus the use of ICT as a diagnostic tool could be employed in a mass survey for lymphatic filariasis. This study has provided a rapid analysis of the situation of the disease in the study area, hence urgent steps need to be taken to implement lymphatic filariasis control programme in the entire Taraba State.

\section{Conflict of Interests}

The authors declare that there is no conflict of interest regarding the publication of this paper.

\section{Acknowledgement}

This study received full sponsorship from Taraba State University, Jalingo through the TETFUND grant. We appreciate and thank the Chairmen and Directors Primary Health Care of Muri Emirate council, Commissioner and Permanent Secretary Ministry of Health, Taraba State for collaborating in carrying out this research project.

\section{References}

1. World Health Organization (1994) Lymphatic filariasis infection and disease: Control strategies, report of a consultative meeting held at the Universiti Sains Malaysia, Penang, Malaysia, August 1994. WHO, Geneva, 52-68.

2. Famakinde DO (2018) Mosquitoes and the lymphatic filarial parasites: Research trends and budding roadmaps to future disease eradication. Trop Med Infect Dis 3: 4.

3. Oparaocha ET, Odaibo AB, Nwoke BEB (2000) Clino-pathological study of human onchocerciasis in Imo river basin, Nigeria and its implication for the control of the disease in the area. Global Journal of Pure and Applied Science 6: 5-9.

4. Usip LP, Opara KN, Ibanga ES, Atting IA, Uttah E (2007) Clinical onchocerciasis in Ini local government area, Akwa Ibom State, Nigeria. Nigerian Journal of Parasitology 27: 36-40.

5. Michael E, Bundy DA, Grenfell BT (1996) Reassessing the global prevalence and distribution of lymphantic filariasis. Parasitology 112: 409-428.

6. Eigege A, Richards Jr FO, Blaney DD, Miri ES, Gontor I, et al. (2002) Rapid assessment for lymphatic filariasis in central Nigeria: A comparison of the immunochromatographic card test and hydrocele rates in an area of high endemicity. Am J Trop Med Hyg 68: 643-646.

7. World Health Organization (1995) Onchocerciasis and its control. Report of a WHO Expert Committee on Onchocerciasis Control. World Health Organ Tech Rep Ser 852: 1-104.

8. Omadu EA, Okafor FC (2007) Rapid epidemiological and socio-cultural appraisal of lymphatic filariasis amongst the Igede ethnic group in Benue State, Nigeria. Nigerian Journal of Parasitology 28: 118-124.

9. World Health Organization (2003) The elimination of lymphatic filariasis. An Interactive guide for Programme Managers. Geneva, 92-120.

10. McMahon JE, Simonsen PE (1996) Filariasis: In Manson's Tropical Diseases, (20th) edn, Cook GC (editor) WB Saunders, London, 1321-1368.

11. Marsden PD (1977) Lymphoraticular filariasis in infectious Disease, a modern treatise of infections processes. 11001102.

12. Schuetz A, Addiss DG, Eberhard ML, Lammie PJ (2000) Evaluation of the whole blood filariasis ICT test for short term monitoring after antifilarial treatment. Am J Trop Med Hyg 62: 502-503.

13. Galvez Tan JZ (2003) The elimination of lymphatic filaria- 
sis: A strategy for poverty alleviation and sustainable development - Perspectives from the Philippines. Filaria J 2: 12.

14. Gyapong JO, Gyapong M, Adjei S (1996) The epidemiology of acute adenolymphangitis (ADL) due to lymphatic filariasis in northern Ghana. Am J Trop Med Hyg 54: 591-595.

15. Nwoke BE, Dozie IN, Jiya J, Saka Y, Okoronkwo C, et al. (2006) The prevalence of hydrocele in Nigeria and its implication on mapping of lymphatic filariasis. Nigerian Journal of Parasitology 27: 29-35.

16. Edungbola LD, Nwoke BEB, Onwuliri CV, Akpa AUC, TayoMafe M (1993) Selection of rapid assessment methods for community diagnosis of onchocerciasis in Nigeria. A recapitulation. Nigerian Journal of Parasitology 14: 3-10.

17. Cheesbrough M (2005) Medical laboratory manual for tropical countries. ELBS/Butter worths kent, 453.

18. Cochrane AL, Holland WW (1971) Validation of screening procedures. Br Med Bull 27: 3-8.

19. Braga C, Dourado MI, Ximenes RA, Alves L, Brayner F, et al. (2003) Field evaluation of the whole blood immunochromatographic test for rapid bancroftian filariasis diagnosis in the northeast of Brazil. Rev Inst Med Trop Sao Paulo 45: 125-129.

20. Iqbal J, Sher A (2006) Determination of the prevalence of lymphatic filariasis among migrant workers in Kuwait by detecting circulating filarial antigen. J Med Microbiol 55: 401405.

21. Targema CN, Onwuliri CO, Mafuyai HB, Mwansat GS, Aida AA, et al. (2008) Mapping of lymphatic filariasis in Benue state, Nigeria. Nigerian Journal of Parasitology 29: 55-61.

22. Ngele KK, Adewale B (2012) A comparative study of the efficiency of Knott's concentration Technique and the immunochromatographic method in the diagnosis of Wuchereria bancrofti. Nigerian Journal of Parasitology 33: 163-175.

23. Norris DE (2004) Mosquito-borne diseases as a consequence of land use change. Eco Health 1: 19-24.
24. Anosike JC, Onwuliri CO, Nwoke BE, Ajayi EG, Oku EE, et al. (2005) Lymphatic filariasis among the Ezza people of Ebonyi State, eastern Nigeria. Ann Agric Environ Med 12: 181-186.

25. Elkanah OS, Elkanah DS, Wahedi JA, Samaila AB, Kela SL, et al. (2017) Lymphatic filariasis in Muri Emirate: Clinical and parasitological studies in Jalingo LGA, Taraba State, Nigeria. Asian Journal of Medicine and Health 6: 1-7.

26. Chandrasena TG, Premaratna R, Abeyewickrema W, de Silva NR (2002) Evaluation of the ICT whole blood antigen card test to detect infection due to Wuchereria bancrofti in Sri Lanka. Trans R Soc Trop Med Hyg 96: 60-63.

27. Omar MS, Sheikah AK, Al-Amari OM, Abdalla SE, Musa RA (2000) Field evaluation of two diagnostic antigen tests for Wuchereria bancrofti infection among Indian expatriates in Saudi Arabia. Southeast Asian J Trop Med Public Health 31: 415-418.

28. Molyneux DH, Taylor MJ (2001) Current status and future prospect of the Global Lymphatic Filariasis programme. Curr Opin Infect Dis 14: 155-159.

29. Sunish IP, Rajendran R, Satyanarayana K, Munirathinam A, Gajanana A (2001) Immunochromatographic test (ICT) for estimation of true prevalence of bancroftian filariasis in an endemic area of Southern India. Trans R Soc Trop Med Hyg 95: 607-609.

30. Koyadun S, Bhumiratana A, Prikchu P (2003) Wuchereria bancrofti antigenaemia clearance among Myanmar migrants after biannual mass treatments with diethylcarbamaxine, $300 \mathrm{mg}$ oral dose FILADEC tablet, in Southern Thailand. Southeast Asian J Trop Med Public Health 34: 758-767.

31. Brooker S, Utzinger J (2007) Integrated disease mapping in a polyparasite world. Geospatial Health 2: 141-146. 